\title{
DETECTOR DE RADIAÇÃO ORGÂNICO E INTELIGENTE PARA RAIOS X DE USO EM RADIOTERAPIA CLÍNICA
}

\author{
Thiago Schimitberger ${ }^{1}$ \\ Giovana Ribeiro Ferreira ${ }^{2}$ \\ Claudiano de Jesus de Souza ${ }^{3}$ \\ Mariana de Mello Silva ${ }^{4}$ \\ Marcelo Frota Saraiva ${ }^{5}$ \\ Rodrigo Fernando Bianchi ${ }^{6}$
}

\section{Resumo}

Neste trabalho são apresentadas a fabricação e a caracterização óptica de um novo detector de raios $X$ para monitoramento da dose nos processos de radioterapia empregando fótons de alta energia (6,0 MV). O dispositivo foi fabricado a partir de materiais orgânicos luminescentes consolidados como elemento ativo de displays eletroluminescentes nanoestrurados, mas que, até esse momento, têm sido pouco investigados como detectores de radiação ionizante. Para tanto, foram preparadas soluções à base de tris(8-hidroxiquinolato) de alumínio - Alq 3 e poli(2-metóxi-5-(2-etilhexiloxi)-pfenilenovinileno) - MEH-PPV, cujo espectro de emissão do primeiro material se sobrepõe ao de absorção do segundo. Os resultados obtidos mostram que os espectros de emissão do sistema $\mathrm{MEH}-\mathrm{PPV} / \mathrm{Alq}_{3}$ desloca-se do vermelho-laranja $\left(\lambda_{\max }=598 \mathrm{~nm}\right)$ para o verde $\left(\lambda_{\max }=545 \mathrm{~nm}\right)$ quando a dose de radiação vai de 0 a $100 \mathrm{~Gy}$. Essa mudança, consequência da degradação induzida pela radiação do MEH-PPV, foi usada no desenvolvimento de dosímetros para possível futura aplicação no controle do tratamento de pacientes oncológicos.

Palavras-chaves: Oncologia; Eletrônica orgânica; Nanotecnologia.

\section{ORGANIC AND SMART RADIATION SENSOR FOR X-RAY IN CLINICAL RADIOTHERPY}

\begin{abstract}
In this work it is presented the characterization and fabrication of a novel ionizing radiation detector for high energy photon beam (6.0 MV). It is used organic luminescent materials usually applied in light-emitting and nanostructure device, but still few explored in radiation dosimetry. Organic solutions of tris(8-hydroxyquinolinato) aluminum - $\mathrm{Alq}_{3}$ and poly[2-methoxy-5-(2'-ethylhexyloxy)-p-phenylene vinylene] - MEH-PPV were prepared to better study the impact of spectral overlap between the $\mathrm{Alq}_{3}$ emission and MEH-PPV absorption. It is observed a blue-shift on the photoluminescence of the MEH-PPV/Alq ${ }_{3}$ solution system from red-orange $\left(\lambda_{\max }=598 \mathrm{~nm}\right)$ to green $\left(\lambda_{\max }=545 \mathrm{~nm}\right)$ when the radiation dose changes from 0 to $100 \mathrm{~Gy}$. This effect is attributed to the photoxidation process of MEH-PPV and was employed to design dose accumulation detector in order to represent easily the radiation dose for cancer treatment.
\end{abstract}

Key words: Cancer; Organic electronic; Nanotechnology.

\footnotetext{
'Doutorando em Ciências e Técnicas Nucleares, Escola de Engenharia, Universidade Federal de Minas Gerais - UFMG, Av. Antônio Carlos, 6627, Campus Pampulha, Cep 31 270-90I, Belo Horizonte, MG. E-mail: tschimitberger@ufmg.br

${ }^{2}$ Aluna de doutorado, Rede Temática de Engenharia de Materiais - REDEMAT, Universidade Federal de Ouro Preto - UFOP,

Campus Universitário Morro do Cruzeiro, Bauxita, Cep 35400-000, Ouro Preto, MG, Brasil. E-mail: giovanaribeiro_ufop@yahoo.com.br

${ }^{3}$ Mestre em Engenharia de Materiais, Rede Temática de Engenharia de Materiais - REDEMAT, Universidade Federal de Ouro Preto - UFOP.

Campus Universitário Morro do Cruzeiro - Bauxita, Cep 35400-000, Ouro Preto, MG, Brasil. E-mail: claudiano.j@gmail.com

${ }^{4}$ Aluna de doutorado, Rede temática de Engenharia de Materiais - REDEMAT, Universidade Federal de Ouro Preto - UFOP,

Campus Universitário Morro do Cruzeiro, Bauxita, Cep 35400-000, Ouro Preto, MG, Brasil.E-mail: mms1985@uol.com.br

${ }^{5}$ Físico Médico, Fundação Cristiano Varella, Hospital do Câncer de Muriaé, Av. Cristiano Ferreira Varella, 555, Bairro Universitário,

Cep 36880-000, Muriaé, MG, Brasil. E-mail: msaraiva@fcr.org.br

${ }^{6}$ Professor Doutor da Universidade Federal de Ouro Preto - UFOP, Campus Universitário Morro do Cruzeiro, Bauxita,

Cep 35400-000, Ouro Preto, MG, Brasil. E-mail: bianchi@iceb.ufop.br
} 


\section{INTRODUÇÃO}

A partir de 1977, com a descoberta das propriedades condutoras do trans-poliacetileno,(1) as moléculas orgânicas conjugadas (polímeros ou cristais orgânicos) tornaram-se o foco de estudo de inúmeros grupos de pesquisa em todo mundo, principalmente, devido aos seus baixos custos de preparação atrelados às facilidades de processamento e de manufatura dos seus dispositivos emissores de luz. ${ }^{(2)}$ Dessa forma, esses polímeros foram considerados como materiais promissores para uso de futuras gerações de monitores, não somente devido o seu fácil processamento e leveza, mas também devido a sua alta luminosidade com baixo consumo de energia. No entanto, mesmo como bons candidatos para aplicações em displays luminosos e outros dispositivos nanoestruturados, a literatura tem apresentado problemas em relação a sua instabilidade sob efeito de radiação. ${ }^{(3)}$ Devido a sua estrutura química com ligações $\pi$-conjugadas, esses materiais são altamente suscetíveis à degradação. Como resultado, esse efeito altera as propriedades ópticas e reduz o tempo de vida (durabilidade) dos seus dispositivos emissores de luz. Todavia, se por um lado o efeito da (foto) degradação constitui uma barreira comercial para os dispositivos emissores de luz orgânicos, ${ }^{(4)}$ por outro, abre a possibilidade de desenvolvimento de detectores de radiação, ${ }^{(5,6)}$ cujas alterações nas propriedades ópticas induzidas pela radiação são mais importantes do que a otimização da durabilidade dos seus dispositivos eletroluminescentes. Em especial, a literatura cientifica vem apontando a necessidade de desenvolvimento de detectores para $\circ$ monitoramento de terapias que envolvem radiação. ${ }^{(7)}$ Neste tipo de terapia, por exemplo, raios $X$ gerados com potencial acelerador de 6,0 MV são muito utilizados no tratamento de pacientes oncológicos. Contudo, superexposição à radiação e outros erros operacionais são problemas frequentes discutidos na imprensa. ${ }^{(7)}$ Neste trabalho, investiga-se o comportamento da fotoluminescência de soluções de poli(2-metóxi,5-etil(2-hexilóxi) parafenilenovinileno) - MEH-PPV e Tris-(8-hidroxiquinolinolato) de alumínio (III) submetidas a doses absorvidas entre 0 - 100 Gy de raios $X$ clínicos (6,0 MV) cujos resultados obtidos apontam para o desenvolvimento de um dispositivo eficiente e inédito para o monitoramento de raios $\mathrm{X}$ (fótons) de alta energia.

\section{MATERIAL E MÉTODOS}

Foram preparadas soluções de tris(8-hydroxiquinolato) de alumínio com concentração de $0,1 \mathrm{mg} / \mathrm{mL}^{(6)}$ e poli[2-metóxi,5- (2'etilhexiloxi)-p- fenilenovinileno] - MEH-PPV dissolvido em clorofórmio $\left(\mathrm{CHCl}_{3}\right)$ com concentração de $0,05 \mathrm{mg} / \mathrm{mL}$, de acordo com os resultados prévios e os procedimentos adotados recentemente por Silva et al. ${ }^{(8)}$ Os materiais foram obtidos da empresa Sigma Aldrich e usados como recebidos. $O$ sistema MEH-PPV/Alq ${ }_{3}$ foi submetido a diferentes doses de raios $\mathrm{X}$, gerados com potencial acelerador de 6,0 MV, oriundos de um acelerador linear Varian Clinac 600 C disponível na Fundação Cristiano Varella, Hospital do Câncer de Muriaé-MG. Durante o procedimento de radiação, soluções com I, $0 \mathrm{~mL}$ foram mantidas em ampolas de vidro, cujas pontas capilares foram lacradas afoticamente para evitar a evaporação do solvente e, consequentemente, evitar mudanças na concentração da solução durante as medidas ópticas. Os sistemas, uma vez preparados, foram colocados em uma caixa de polipropileno (PP) e submersos a uma profundidade de $1,5 \mathrm{~cm}$ para garantir a dose planejada. As placas de PMMA colocadas abaixo do detector garantem as condições de retroespalhamento da radiação (Figura la). As amostras foram alinhadas com
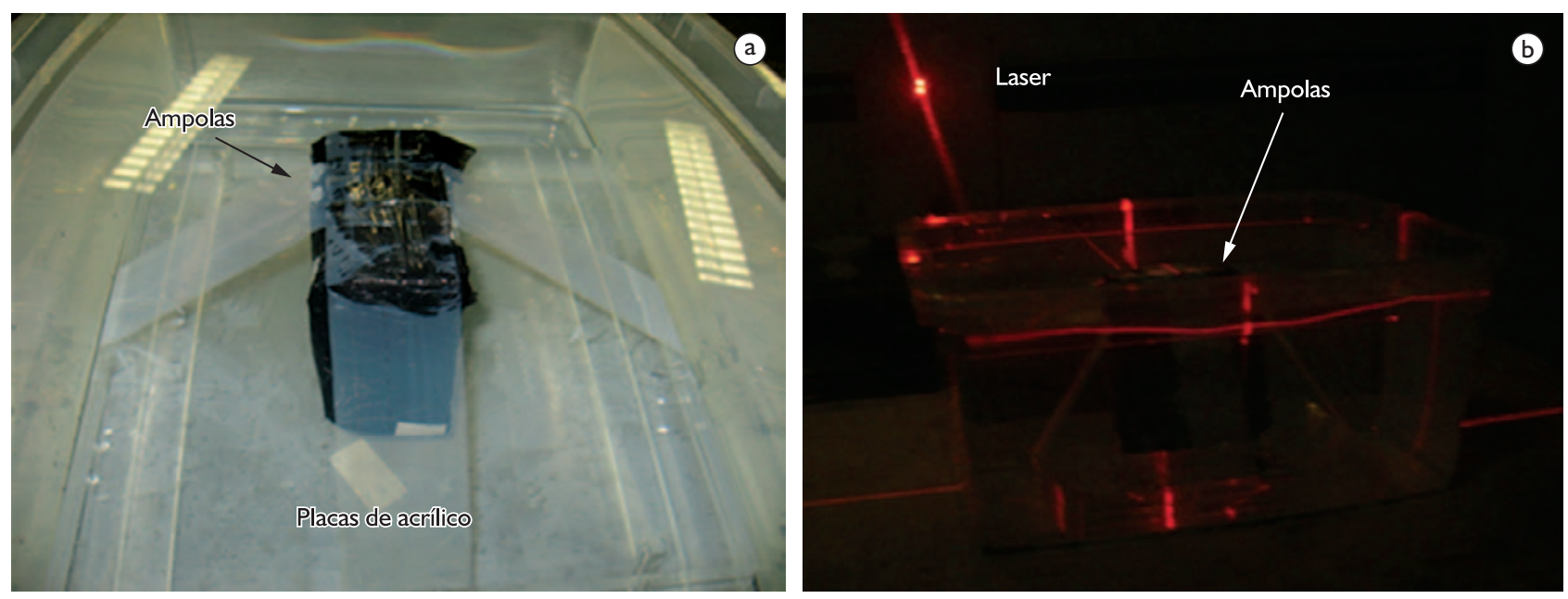

Figura I. Amostras dos materiais orgânicos luminescentes sendo preparadas para a irradiação: a) amostras submersas na água a uma distância mínima de I,5 cm entre as ampolas e a superfície da água, e sobre placas de PMMA, bom simulador para o tecido mole; b) amostras sendo ajustadas com ajuda de lasers para garantir que o campo de irradiação esteja a I,0 m de distância da superfície da água e que o campo de radiação incida em todas as amostras. 
ajuda de lasers (Figura Ib) mantendo-se a uma distância de I,0 $\mathrm{m}$ entre a superfície da água e o acelerador; consequentemente, foram expostas a diferentes doses de raios $X$ (fótons) entre 0 a 100 Gy e caracterizadas por meio de medidas de espectroscopia de absorção na região do ultravioleta visível (UV-VIS), empregando um espectrofotômetro UV-VIS Shimadzu série I650, e de fotoemissão, empregando um espectrofotômetro Ocean Optics USB650, cuja excitação foi realizada com LED violeta $\left(\lambda_{\text {máx }}=405 \mathrm{~nm}\right)$ de baixa intensidade.

\section{RESULTADOS}

A Figura 2 mostra os espectros de absorção e de fotoluminescência de soluções de MEH-PPV e $\mathrm{Alq}_{3}$ (a) antes e (b) depois da exposição à radiação ( $100 \mathrm{~Gy}$ ). Nos resultados apresentados mostrados nessa figura, observa-se que não há mudanças nas propriedades ópticas do $\mathrm{Alq}_{3} \mathrm{com}$ a exposição à radiação; mas, por outro lado, deslocamentos para comprimentos de onda menores e a diminuição das intensidades dos espectros são observados para o MEH-PPV. Esses materiais foram estudados separadamente para avaliar e correlacionar a região de sobreposição dos espectros de absorção e de emissão desses sistemas, com o objetivo de propor um sistema que tenha sua emissão máxima variada com a exposição à radiação, ou seja, que emita inicialmente no vermelho e passe a emitir na região do verde à medida que a dose de radiação aumenta. ${ }^{(9)}$ Nesse sentido, a Figura 3 apresenta os espectros de fotoluminescência obtidos com a solução de $\mathrm{MEH}-\mathrm{PPV} / \mathrm{Alq}_{3}$ exposta a doses de radiação variando de 0 a $100 \mathrm{~Gy}$. Como pode ser observado, a radiação atua no sentido de diminuir a contribuição da emissão do MEH-PPV e aumentar a do $\mathrm{Alq}_{3}$ nos sistemas investigados. Essas alterações mudam drasticamente as propriedades ópticas dos filmes de $\mathrm{MEH}-\mathrm{PPV} / \mathrm{Alq}_{3}$, deslocando o espectro de fotoluminescência do filme do vermelho ao verde, como era esperado. Em resumo, observa-se na Figura 3 que as soluções não expostas à radiação apresentam espectro de fotoemissão típico do MEH-PPV, de coloração vermelho-laranja com $\lambda_{\text {máx }} \sim 570 \mathrm{~nm}$. Entretanto, após a exposição do sistema para doses mais altas de radiação (100 Gy), a mistura polímero-cristal orgânico passa a emitir na região do verde, que é uma característica do $\mathrm{Alq}_{3}$ (Figura 2). No entanto, a cor da radiação emitida não é determinada apenas pelo comprimento de onda máximo e sim por todo o espectro de fotoluminescência. Portanto, a partir das coordenadas de cores virtuais $\mathrm{X}, \mathrm{Y}$ e $\mathrm{Z}$ fornecidas pelo espectrofotômetro são calculadas as coordenadas de cromáticas $x$ e $y$. $O$ diagrama de cromaticidade é apresentado na Figura 4.

$\mathrm{Na}$ Figura 4, observa-se claramente que a solução orgânica excitada muda do vermelho ao verde com a exposição à radiação. Isso é corroborado pela análise direta de

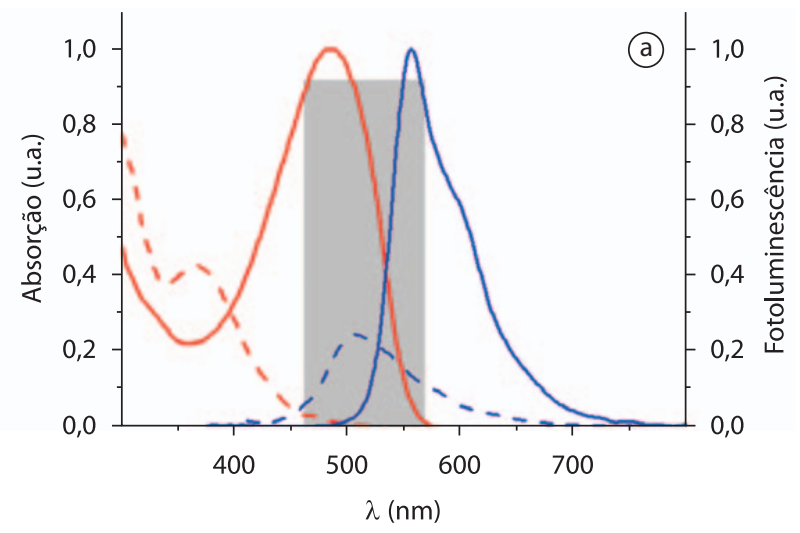

Espectro de absorção (MEH-PP)

- Não irradiado

Espectro de fotoluminescência (MEH-PPV)

- Não irradiado

- - - - - Irradiado (100 Gy)
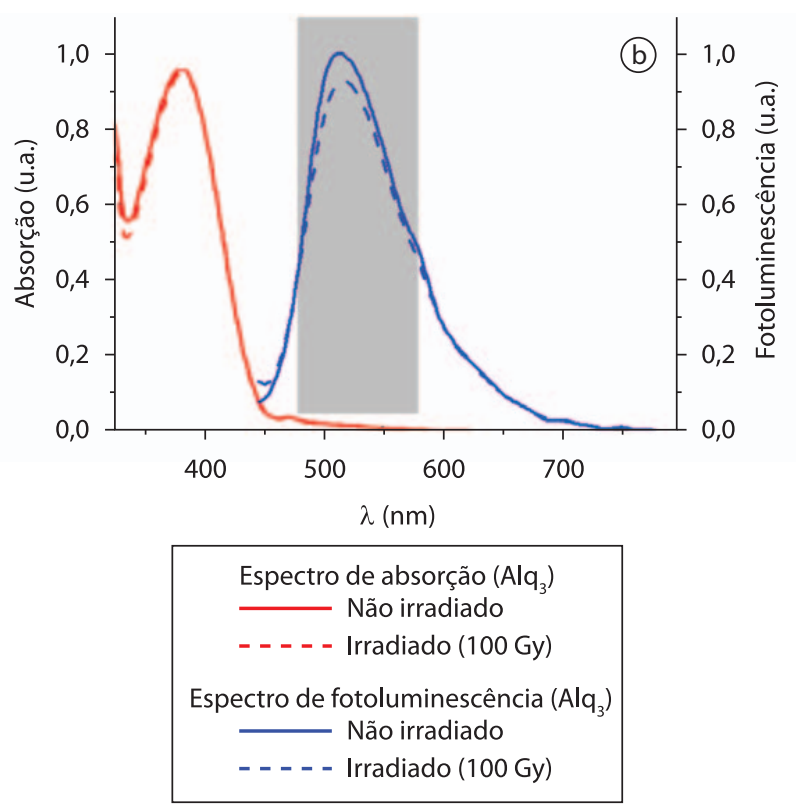

Figura 2. a) Espectros de absorção e fotoluminescência de soluções de: a) $\mathrm{MEH}-\mathrm{PPV}$ e b) $\mathrm{Alq}_{3}$, não irradiados e depois de receberem $100 \mathrm{~Gy}$.

soluções de MEH-PPV/Alq ${ }_{3}$ expostas a diferentes doses de radiação e excitadas com luz azul $(430 \mathrm{~nm}-460 \mathrm{~nm})$. Os resultados obtidos são apresentados na Figura 5.

\section{DISCUSSÃO}

$\mathrm{O}$ uso de MEH-PPV e $\mathrm{Alq}_{3}$ como elemento ativo de detectores de radiação é ainda muito recente na literatura e não deixa dúvidas quanto à atualidade desse tema para desenvolvimento de sensores de radiação inéditos e eficientes. Ademais, os poucos resultados existentes ${ }^{(6,9)}$ 


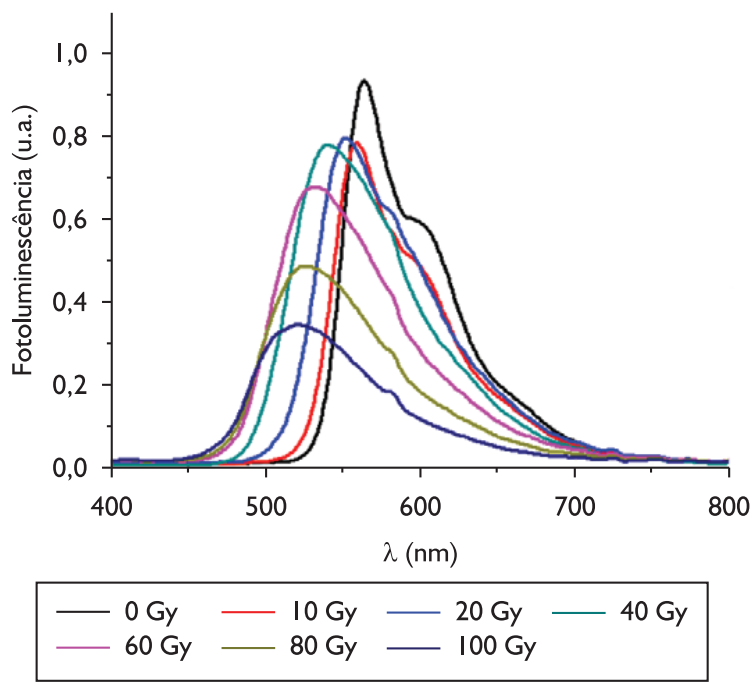

Figura 3. Espectro de fotoluminescência de soluções de MEHPPV/ Alq3 exposta à diferentes doses.

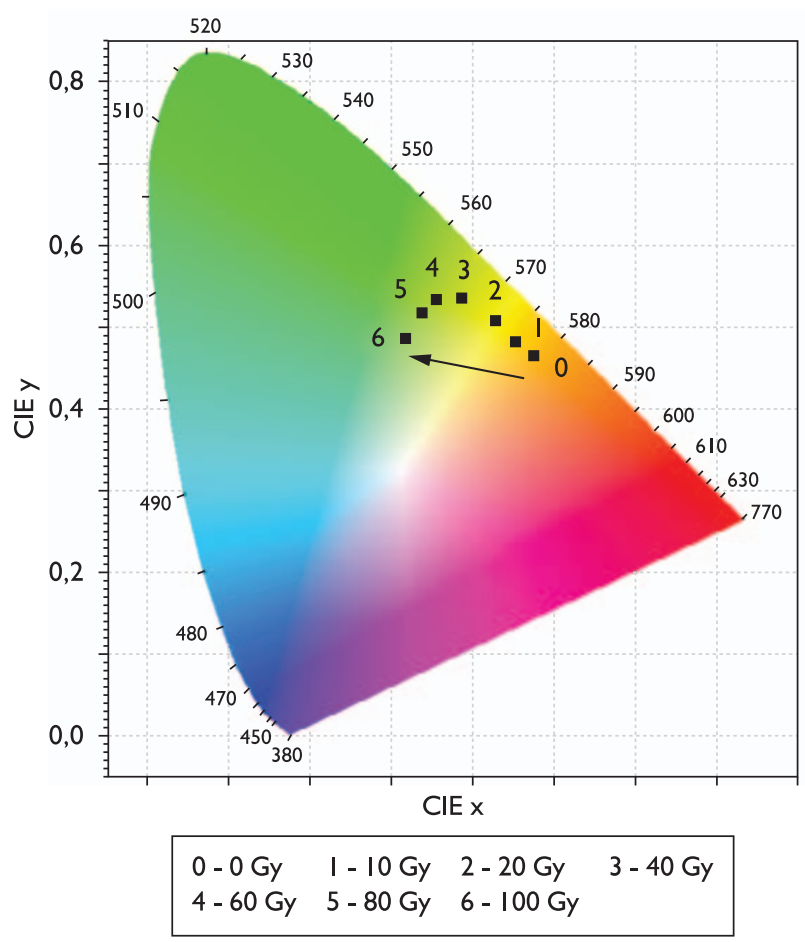

Figura 4. Diagrama de cromaticidade representando a mudança de cores apresentada pela solução quando excitadas com um LED violeta $\left(\lambda_{\text {máx }}=405 \mathrm{~nm}\right)$ exposta à doses de $0 \mathrm{~Gy}, 10 \mathrm{~Gy}, 20 \mathrm{~Gy}, 40 \mathrm{~Gy}$, $60 \mathrm{~Gy}, 80 \mathrm{~Gy}$ e $100 \mathrm{~Gy}$ de fótons de raios X clínicos (6,0 MV).

foram obtidos expondo esses sistemas à radiação visível e, portanto, não há relato do uso desses dois materiais para uso como detectores de radiação ionizante, tampouco para raios $X$ (fótons) e alta energia. Sabe-se que a radiação interage com o solvente (clorofórmio) liberando, na solução, radicais livres $\left(\mathrm{Cl}^{-}\right)$. Os radicais livres são espécies químicas instáveis que facilitam os processos oxidativos que estão vinculados aos processos de degradação do

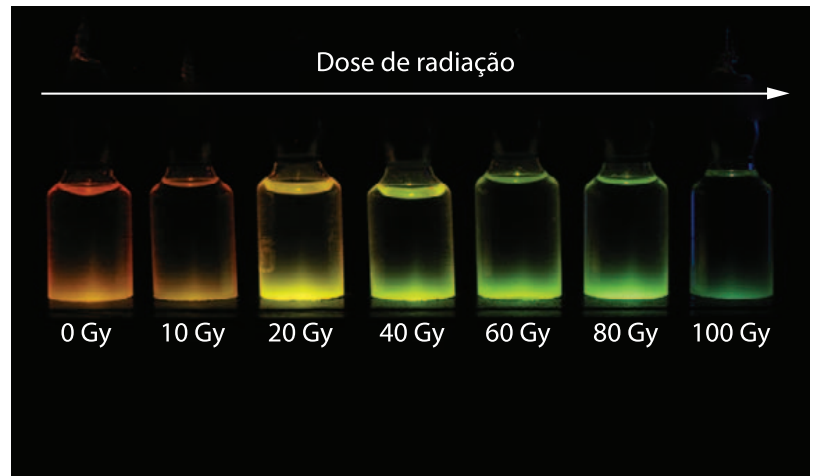

Figura 5. Sistema constituído pelas ampolas de vidro preenchidas com o sistema híbrido soluções do polímero luminescente e do cristal orgânico submetidas a diferentes doses exposição à radiação.

MEH-PPV. Logo, à medida que as soluções são expostas à radiação existe a alta taxa de liberação de radicais $\mathrm{Cl}^{-}$, que aumenta a degradação dos sistemas orgânicos luminescentes, alterando drasticamente as propriedades ópticas dos sistemas.

Devido aos altos índices de overdose ocorrido no mundo para tratamento de câncer proveniente de aceleradores lineares - problema que, na maioria das vezes, vem ocorrendo principalmente por erros operacionais $^{(7)}$ - é possível, com a evolução desses sistemas, utilizá-los como detectores em tempo real empregados junto aos pacientes oncológicos durante o tratamento radioterápico para um melhor controle desses tratamentos. Este é o foco deste trabalho: propor uma solução para um problema operacional baseado na resposta óptica de materiais bem consolidados como elemento ativo de dispositivos eletroluminescentes, mas pouco explorados como sensores de radiação.

\section{CONCLUSÃO}

Os resultados apresentados neste trabalho mostram, pela primeira vez na literatura, que soluções orgânicas baseadas em cristal orgânico luminescente, $\mathrm{Alq}_{3}$, e um polímero cujas propriedades ópticas são alteradas pela radiação, o MEH-PPV, são candidatos potenciais para $\circ$ monitoramento e controle dos tratamentos de pacientes oncológicos via radioterapia com raios $X$ (fótons clínicos). As vantagens desse sistema são: facilidade de leitura de dose, facilidade de processamento e baixo custo $(<\mathrm{R} \$ \mathrm{I}, 00)$.

\section{Agradecimentos}

Os autores agradecem à Fundação Cristiano Varella - Hospital do Câncer de Muriaé - MG pela utilização do Acelerador Linear, e ao INEO/CNPq, à Capes, ao CNPq e à Fapemig pelo suporte financeiro. 


\section{REFERÊNCIAS}

I CHIANG, C. K. et al. Electrical conductivity in doped polyacetylene. Physical Review Letters, v. 39, n. 17, p. 1098101, 1997. http://dx.doi.org/10.1 103/PhysRevLett.39.1098

2 NALWA, H. S. Organic conductive molecules and polymers. New York: John Wiley \& Sons, 1997.

3 BIANCHI, R. F. et al. Ellipsometry study of the photo-oxidation of poly[(2-methoxy-5-hexyloxy)-pphenylenevinylene]. Journal of Polymer Science Part B: Polymer Physics, v. 42, n. 6, p.1033-4I, Mar. 2004. http://dx.doi. org/10.1002/polb. 10734

4 FOROOHAR, R. One word: plastics: the next big thing in electronics might be talking magazine ads, smart spray paint and foldable displays. Newsweek International, 23 Feb. 2004: 54. Academic Onefile. Web. 29 Jan, 2010.

5 VASCONCELOS; K. B.; BIANCHI, R. F. Polímeros luminescentes como sensores de radiação não ionizante: aplicação em fototerapia neonatal. Polímeros, v. 17, n. 4, p. 325-8, Maio 2007.

6 FERREIRA, G. R. C.; VASCONCELOS, K. B.; BIANCHI, R. F. Design and characterization of a novel indicator dosimeter for blue-light radiation. Medical Physics, v. 36, n. 2, p. 642-4, Jan. 2009. PMid:19292005. http://dx.doi. org/10.1 II 18/1.3039788

7 BOGDANICH, W. The radiation boom: radiation offers new cures, and ways to do harm. The New York Times, 24 jan. 2010. Disponivel em: <http://www.nytimes.com/20l0/0I/24/health/24radiation.htmL>. Acesso em: mar. 2010.

8 SILVA, E. A. B. et al. Low dose ionizing radiation detection using conjugated polymers. Applied Physics Letters, v. 86, n. 2, p. I31902-3, Mar. 2005. http://dx.doi.org/10.1063/I.189/300

9 VASCONCELOS, C. K. B.; BIANCHI, R. F. A blue-light dosimeter which indicates the dose accumulation by a multicoloured change of photodegraded polymer. Sensors and Actuators. B, Chemical, v. I43, n. I, p. 30-4, 19 Sep. 2009. http://dx.doi.org/10.1016/j.snb.2009.09.009

Recebido em: $25 / 08 / 201$ I

Aceito em: $15 / 01 / 2012$ 